\title{
The Role of Palliative Care in the Management of Patients with Advanced Hepatocellular Carcinoma: A Single Institution Experience
}

\section{Ayman $\mathrm{A}^{1}$, Azza $\mathrm{AH}^{1,2 *}$, Yasser $\mathrm{K}^{3,4}$, Kakil $\mathbf{R}^{1}$, Jonas $\mathrm{F}^{1}$, Asma Mohammed $\mathrm{AB}^{1}$ and Zeinab $\mathbf{M I}^{1}$}

${ }^{1}$ National Center for Cancer Care and Research, HMC, Doha, Qatar

${ }^{2}$ Cancer Management and Research, Medical Research Institute, Alexandria University, Egypt

${ }^{3}$ Medicine Department, Gastroenterology and Hepatology Section, HMC, Doha, Qatar

${ }^{4}$ Medicine Department, Minia University, Egypt

\section{Abstract}

Introduction: Advanced HCC at initial presentation is as high as $15 \%$. Those patients present with a complex group of symptoms that need to be addressed to improve their quality of life.

Objective: Analyze the characteristics of patients with advanced HCC and determine their need for Supportive and Palliative care.

Methods: The current study included a retrospective review of all patients with advanced HCC accepted under the palliative care program at the NCCCR, Doha, Qatar from 2012 till 2015. A detailed descriptive analysis of those patients was performed.

Results: 40 patients constituted the cohort of this study. Ratio was $3: 1.75 \%$ of patients were above 60 years. Previous HBV or HCV was present in $95 \%$ of patients. Most common symptoms were fatigue, pain, anorexia, drowsiness, anxiety and depression. Incidence of advanced HCC was $25 \%$ of all HCC cases and $11 \%$ of all cases accepted under the palliative care program in the past 4 years. Ascites was present in $80 \%$ of patients, and hepatic encephalopathy in $82 \%$ of patients. Distant metastases (to bones and lungs) occurred in $45 \%$ of patients. The rest of patients (55\%) had multifocal liver lesions. Median OS was 7 months. Majority of our patients (94\%) died in the hospital.

Conclusion: Advanced HCC is as common as $25 \%$ at initial presentation. Patients with such diagnosis present with symptoms that would require early engagement with aspecialized palliative care program to address those symptoms. The aim of treatment for those groups of patients should be solely to improve their quality of life.

Keywords: Advanced HCC; Supportive care; Palliative care; Symptom management; Symptom prevalance; Quality of life

\section{Introduction}

Hepatocellular Carcinoma (HCC) constitutes the fifth most common malignancy in adults [1]. The incidence is highest in Africa and Asia where it is correlated to Hepatitis B virus (HBV) infection and to a lesser extent hepatitis $\mathrm{C}$ virus (HCV) infection $[2,3]$. Unlike Asia where hepatitis $\mathrm{B}$ virus infection is the predominant infection strongly associated with the subsequent development of Hepatocellular Carcinoma, hepatitis $\mathrm{C}$ virus infection is much more common as a predisposing factor for development of HCC in Africa and the Middle East [4].

A recent study done in Egypt, reported an overall incidence of chronic HCV infection of $9.1 \%$ with an all-cause mortality rate attributable to chronic HCV infection of $5.7 \%$, while the liver-related mortality was $45.5 \%$ [5].

Among HCC patients, $15-20 \%$ present with advanced HCC at initial diagnosis globally [4-8]. This advanced presentation is usually associated with severe physical symptoms including pain, fatigue, generalized weakness, anorexia as well as psychosocial distress [8]. Those symptoms have a negative impact on Quality of Life (QOL) and functional status of patients with advanced HCC. The definition of end stage or advanced HCC applies to patients with tumors leading to a very poor performance status (ECOG 3-4) or Child-Pugh C patients who are not amenable to transplantation $[1,2,4]$.

The median survival of such patients is less than 6 months, with a 1 year survival of $11 \%[9,10]$. As a result of this dismal survival, the management of patients with advanced HCC is only symptomatic and no definitive tumor directed therapy is indicated. Those patients should receive only palliative supportive treatment aiming to improve their QOL. In general, they should not be considered for participating in clinical trials [4]. The treatment for those cases should be addressed towards the management of pain and non-pain symptom, nutrition and psychosocial support. The aim of the present study is to review our own experience with patients diagnosed with advanced HCC who have been accepted under the palliative care program at the National Center for Cancer care and Research (NCCCR) in the state of Qatar over the past 4 years (from 2012 till 2015).

\section{Materials and Methods}

The charts of patients with the diagnosis of advanced HCC accepted under the Palliative care program at National Center for Cancer Care and Research (NCCCR) between 01/01/2012 till 31/12/2015 were

*Corresponding author: Azza Adel Hassan, National Center for Cancer Care and Research, HMC, Doha, Qatar, Tel: 0097466578952; Fax: 0044397857;

E-mail: newazza@gmail.com

Received: May 15, 2016; Accepted: May 31, 2016; Published: June 07, 2016

Citation: Ayman A, Azza AH, Yasser K, Kakil R, Jonas F, et al. (2016) The Role of Palliative Care in the Management of Patients with Advanced Hepatocellula Carcinoma: A Single Institution Experience. J Pat Care 2: 112. doi: 10.4172/2573 4598.1000112

Copyright: $\odot 2016$ Ayman A, et al. This is an open-access article distributed unde the terms of the Creative Commons Attribution License, which permits unrestricted use, distribution, and reproduction in any medium, provided the original author and source are credited. 
Citation: Ayman A, Azza AH, Yasser K, Kakil R, Jonas F, et al. (2016) The Role of Palliative Care in the Management of Patients with Advanced Hepatocellular Carcinoma: A Single Institution Experience. J Pat Care 2: 112. doi: 10.4172/2573-4598.1000112

reviewed. The parameters analyzed were age, gender, nationality, site of metastases, most common presenting symptoms, site and type of pain, presence or absence of hepatic encephalopathy, and or ascites. The review also included previous history of hepatitis B or Hepatitis $\mathrm{C}$ associated infection, presence of liver cirrhosis as well as a detailed history of associated co-morbid conditions (Diabetes, hypertension, cardiac and renal). Their coagulation profile was also reviewed (normal or deranged). Presence or absence of hypoalbunemia was also reported, (See Table 1 for patients' characteristics).

Treatment details including Surgery, chemotherapy, targeted therapy (Sorafinib) and Radiotherapy were also analyzed. A direct survival analysis was also reported for this cohort of patients. The place of death (Palliative care unit, home or Hamad General Hospital) for patients who expired as a result of their disease were studied. The aim of treatment in those cohorts of patients was to control their symptoms for the remainder of their life. No additional survival advantage could be attributed to this symptom-directed therapy.

\section{Results}

A total of 40 patients with the diagnosis of advanced HCC (ECOG 3-4 or Child-Pugh C) were accepted under the palliative care program at NCCCR in the last 4 years, those constituted the cohort of the present study. HCC represented $11 \%$ of the patients completely transferred to the palliative care unit (40/363 patients) during the study period and $25 \%$ (40/160\% patients) of all cases of HCC who presented to Hamad General Hospital during the same period of time. There were

\begin{tabular}{|l|c|c|}
\hline \multicolumn{2}{|c|}{} & No. of patients \\
\hline \multirow{2}{*}{ Gender } & Male & $30(75 \%)$ \\
\hline \multirow{2}{*}{ 2. Age } & Female & $10(25 \%)$ \\
\hline \multirow{2}{*}{ 3.Nationality } & $>60$ years & $30(75 \%)$ \\
\hline \multirow{2}{*}{ 4.Presence of HBV or } & $<60$ years & $10(25 \%)$ \\
\hline HCV Infection & Qatari & $8(20 \%)$ \\
\hline \multirow{2}{*}{ 5. Presence of Liver Cirrhosis } & Non-Qatari* & $32(80 \%)$ \\
\cline { 2 - 3 } & Yes & $38(95 \%)$ \\
\cline { 2 - 3 } & No & $2(5 \%)$ \\
\hline \multirow{2}{*}{ Non } & Yes & $33(83 \%)$ \\
\hline
\end{tabular}

*Non-Qatari patients were as follows: Egyptians 11 patients, Pakistanis 7 patients, Jordanian 4 patients, Yemeni 3 patients, Saudi Arabia 2 patients, and 1 patient from each of the following countries Emirates, Mauritania, Oman, UK and Canada Table1: Characteristics of 40 included patients.
30 males $(75 \%)$ and 10 females (25\%) with a ratio of 3:1. The nonQatari patients were much more common (32 patients) than the Qatari patients (8 patients). Patients 60 years old or older represented $75 \%$ of the population while only $25 \%$ of patients were younger than 60 years of age. History of Hepatitis B or C virus infection was quite common among the studied patients (95\%). Also, patients presenting with hepatic encephalopathy were more common (83\%), $80 \%$ of patients presented with ascites and $83 \%$ had a background of associated liver cirrhosis. Nearly half of the patients $18 / 40$ patients (45\%) presented with metastatic disease outside the liver, including bone and lung metastases as well as other odd sites of metastases such as skin and subcutaneous sites and spinal cord compression at initial presentation. About $45 \%$ of patients presented with multifocal liver lesions. The most frequently encountered symptoms included tiredness (100\%), anorexia (89\%), pain (82\%), anxiety (55\%), drowsiness (53\%), depression $(47 \%)$, shortness of breath (34\%) and nausea (26\%), (See Figure 1 and Table 2, for most common presenting symptoms). Dull-aching abdominal pain was the most common type of pain encountered (78\%), followed by sharp like pain $(22 \%)$. The mean severity of pain was around 3.4 out of 10 on the Numerical Rating Scale (NRS).

The most common associated co-morbidities were hepatic disease (87\%), hypertension (63\%) followed by diabetes mellitus (58\%). Fewer patients had associated renal (24\%) or cardiac diseases $(21 \%)$. The overall well-being of patients (i.e., QOL) was affected by the disease in the majority of patients (84\%). Treatments previously received by patients prior to their acceptance under palliative care included palliative surgical intervention (14\%), palliative chemotherapy, (Tran arterial-Doxorubicin based chemoembolization (TACE) and targeted therapy (Mainly 2-3 cycles of Sorafinib) in $48 \%$ of patients as well as palliative radiotherapy (21\%) to metastatic bony lesions and brain

\begin{tabular}{|l|c|c|}
\hline Symptoms & No of Patients & Percentage \\
\hline 1. Fatigue & 40 & $100 \%$ \\
\hline 2. Anorexia & 36 & $89 \%$ \\
\hline 3. Pain (Mainly abdominal pain) & 33 & $82 \%$ \\
\hline 4. Anxiety & 22 & $55 \%$ \\
\hline 5. Drowsiness & 21 & $53 \%$ \\
\hline 6. Depression & 19 & $47 \%$ \\
\hline 7. Shortness of Breath & 14 & $34 \%$ \\
\hline
\end{tabular}

Table 2: Percentage of the most common presenting symptoms among the 40 patients.

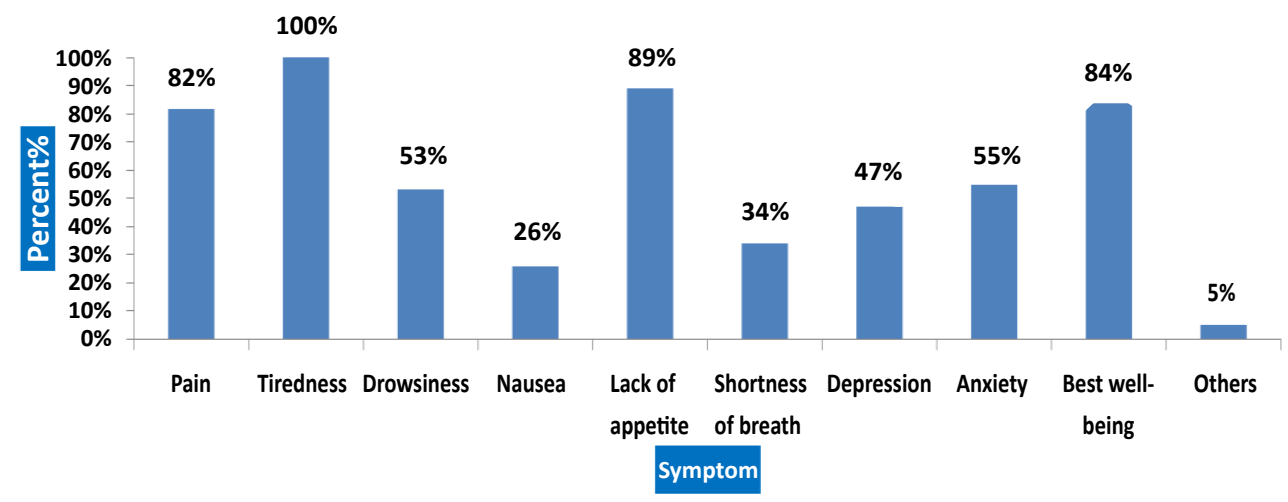

Figure 1: Distribution of symptoms among included patients. 
metastases. The aim of all of those treatments was to prolong the disease-progression interval. The most common strong opioid used for the treatment of pain was Morphine in 30 patients (75\%), followed by Fentanyl patches in 7 patients (18\%). It is worth noting that all patients treated with fentanyl transdermal patches had morphine immediate release for breakthrough pain. Methadone was used only in 3 patients, one of them had refractory pain to both morphine and fentanyl. Tramadol was also used in 3 patients. Midazolam (in 7 patients) and Haloperidol (in 6 patients) were both used in the treatment of associated hyperactive delirium. The median overall survival for the whole cohort of patients was 7 months with a range from 1.8 months to 88.1 months (Figure 2). A total of 35 patients (88\%) died as a result of progressive advanced HCC, while 5 patients (12\%) are alive with advanced disease. The majority of deaths occurred in the palliative care unit $(91 \%)$, while $6 \%$ died at home and $3 \%$ died at Hamad General Hospital. Figure 3 represents a comparative survival according to gender while Figure 4 represents comparative survival according to age ( $\leq 60$ years versus $>60$ years).

\section{Discussion}

The present study clearly shows that advanced HCC is not an uncommon presentation at our Cancer Center, representing $11 \%$ of all patients accepted under the palliative care program and $25 \%$ of all HCC

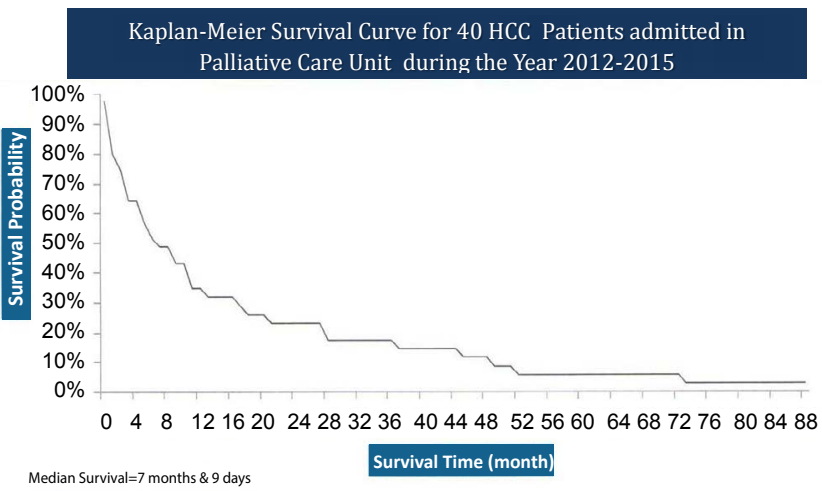

Figure 2: Overall median survival for the whole cohort.

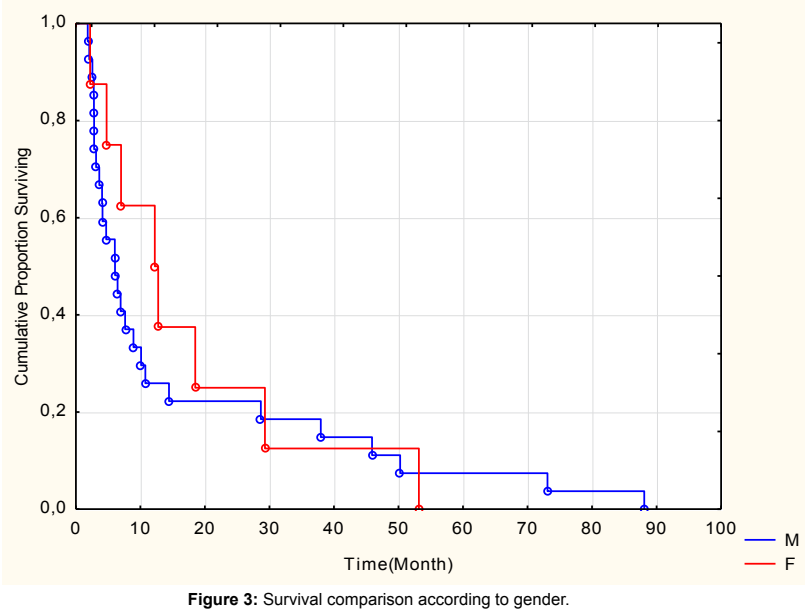

$\mathrm{M}=$ Males, $\mathrm{F}=$ Females

Figure 3: Survival comparison according to gender.

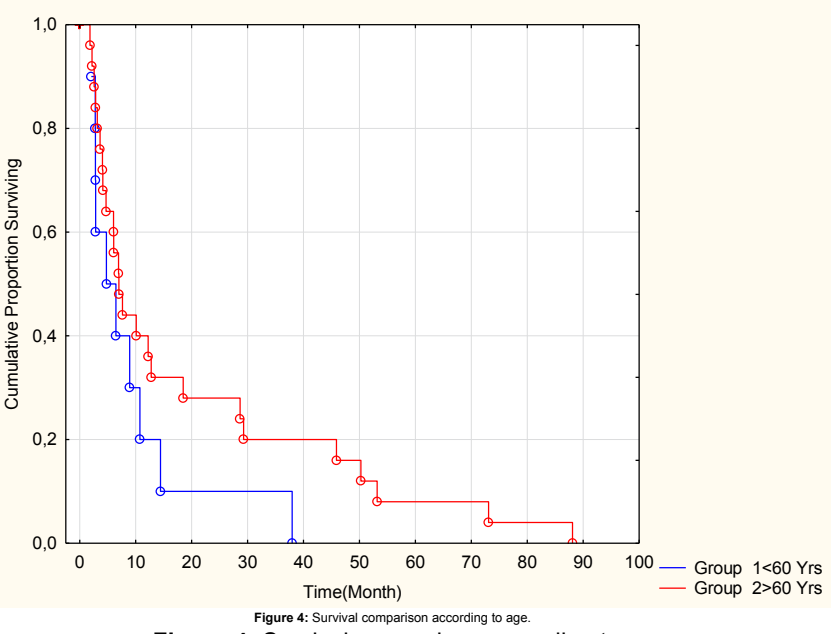

Figure 4: Survival comparison according to age.

presenting to Hamad Medical Corporation in the last 4 years (from 1-12012 till 31-12-2015). Those figures constitute the national figures of the State of Qatar, since Hamad Medical Corporation is the only nonprofit organization accepting patients with HCC in Qatar [11]. Those figures are in agreement with most of the published literature related to the incidence of advanced HCC [9,12-14].

The explicit review of the most common presenting symptoms among our patient's revealed fatigue and tiredness as well as pain to be the most common symptoms ( $100 \%$ and $80 \%$ respectively). Further analysis of pain characteristics revealed a dull-aching abdominal pain to be the most common type of pain experienced by our cohort of patients $(78 \%)$, although not severe on NRS $[3,4]$. This is again in conjunction with all the published literature in this aspect [4,15-17].

Lack of Appetite has not been less frequent as a presenting symptom compared to fatigue and pain in the present study (89\%). This illustrates the challenge of malnutrition imposed by such an advanced disease, and highlights the importance of addressing the difficult problem of anorexia-cachexia syndrome and to what extent one should try to improve the nutritional status of such patients. The balance between adequate nutrition of a patient with advanced HCC versus a situation where adequate nutrition becomes of a lesser priority (e.g. in patients at the end of their life) depends on accurate prognostication both for Oncologist and palliative care physicians. There are scenarios in which insisting on adequate nutrition becomes an unnecessary burden (e.g. in patients with less than 1-2 weeks of life) that impairs their remainder survival as well as their quality of life [18]. It is the undoubted right of patients and their caregivers to be informed about such situations [1820]. The present study also clearly demonstrates the strong association of previous HBV as well as HCV infections with the subsequent development of HCC (95\% association). It is well known that the viral induced chronic liver disease is a common risk factor all over the world for HCC with regional variations [21].

In a previous study, the incidence of HBV among patients with liver disease in Qatar was found to be relatively rare in comparison to a much higher incidence of HCV (2.5\% vs. $29 \%$ respectively) [22]. This higher incidence of HCV prevalence in Qatar is emphasized by the fact of a growing expatriate worker population from high prevalence region with HCV infection like Egypt and Pakistan. The fact that HCV and HBV are the underlying etiology for the development of HCC in Qatar 
was mentioned by Rasul et al. [23]. Also, the presence of a background of liver cirrhosis in the present study has been high (83\%), this is in accordance with most of the published literature [24]. The associated liver cirrhosis renders the choice of strong opioids for the treatment of associated pain even more challenging. It is well known that Morphine and to a greater extent Methadone (which are mainly metabolized in liver) are not the first choice of opioids especially in severe hepatic impairment. In such situations, Fentanyl (given as a Transdermal patch or parenterally through a continuous subcutaneous or intravenous infusion) becomes the first choice especially in cases of hepatorenal syndrome $[4,25]$.

An altered fluctuating level of consciousness (LOC) as a result of associated variable degrees of hepatic encephalopathy is to be expected during the course of this disease. This alteration in LOC could be improved in the early stages of the disease through the use of Lactulose syrup or enemas, and assuring 2-3 adequate bowel movements per day $[26,27]$. However, hepatic encephalopathy with an associated delirium and impaired LOC becomes irreversible in the terminal stages of the disease $[28,29]$. This symptom was common in the present study $(83 \%$ of patients). Haloperidol (a neuroleptic antipsychotic medication) has been proven to be beneficial for patients with advanced HCC with associated delirium, whether hypoactive or hyperactive. It has also the advantage of being non-sedative [30].

The management of an associated ascites is variable among different centers. Usually, ascitic tapping (an average of 3-4 L/day) is performed in patients who develop symptoms from ascites such as abdominal pain, nausea, vomiting, shortness of breath or anorexia resulting from considerable stretching abdominal distension. The use of replacement parenteral human albumin is usually added to ascitic tapping, although it did not demonstrate a long-term efficacy. The use of double diuretic therapy (Furosemide+Spironolactone) has been largely tried to delay for as long as possible the re-accumulation of ascites, and the need for repeated tapping [31]. The incidence of ascites has been reported to be as high as $80 \%$ in the present study. All the patients included in our study had a poor performance score (ECOG 3-4), and were child-Pugh type $\mathrm{C}$, with the majority of patients $(75 \%)$ above the age of 60 years. We believe as others that this category of patients should not be included in any experimental clinical trial and that the focus of their management should be on symptom management and improvement of the quality of their remainder life [4].

It is of interest to notice that the associated psychosocial distress among patients with advanced HCC is often overlooked. In fact, in previous publications, HCC ranked third among the most common 14 types of cancer associated with psychosocial distress [8,32]. In our study, although patients were not screened for psychosocial distress, the incidence of depression (47\%) as well as anxiety (55\%) were high. This illustrates the importance of initial screening for psychosocial distress for patients with advanced HCC as soon as they are accepted under the palliative care program. Currently, we are performing a prospective trial using the distress thermometer to evaluate psychosocial distress for patients with different cancer diagnosis at different stages of their disease trajectory (work in progress).

The incidence of advanced HCC at NCCCR represented 25\% of all HCC patients seen at Hamad Medical Corporation in the last 4 years. This is in accordance with international figures published in the literature [4]. The overall median survival of our cohort of patients was 7 months (Figure 2), which is slightly higher than the figure most commonly reported literature ranging from 3-6 months. This entails that an early engagement with a specialized supportive and palliative care program is warranted for such patients aiming to improve their symptoms and hence their quality of life (in terms of self-care, alertness, social interactions, nutritional needs through eating as well as ambulation). It also stresses that patients with such dismal prognosis should not be subjected to any experimental tumor directed therapy or participates in any clinical trial involving investigational therapy.

Finally, the fact that $94 \%$ of the patients died in the hospital (whether in the Palliative care unit or at Hamad General Hospital) while only $6 \%$ died at home, has been previously demonstrated in a previous publication from our center [33]. This pattern could be attributed to the lack of a well-developed community palliative care service that can support the needs of patients and their families while at home. Efforts and plans have started to develop such service for patients with advanced cancer diagnosis in the State of Qatar.

\section{Summary and Conclusion}

In summary, the incidence of advanced HCC is not uncommon among the palliative care population. Those patients present with a challenging complex spectrum of physical and non-physical symptoms that should be addressed as early as possible along the disease trajectory. The aim of treatment for such patients should be focused on symptom control as well as on improvement in QOL. Those patients should not participate in any of the clinical trials that are essentially tumordirected therapies. Most of our patients have a background of liver cirrhosis with a variable degree of hepatic impairment, which makes the choice of appropriate opioid medications more challenging. There is an undoubted need to initially assess those patients for the associated psychosocial distress which has been already shown to be of high prevalence in such disease. Despite the dismal survival of such patients, yet they have a median survival around 7 months, which renders early integration with a specialized palliative care service an important goal to improve the quality of life of those patients and maximize their symptom control (Tables 1 and 2) (Figures 1-4).

\section{References}

1. Mc Cracken M, Olsen M, Chen Jr MS, Jemal A, Thun M, et al. (2007) Cancer incidence, mortality, and associated risk factors among Asian Americans of Chinese, Filipino, Vietnamese, Korean and Japanese ethnicities. CA Cancer Clin 57:190-205

2. Park Y (2002) M Hepatocellular carcinoma in Asia. In: Saria SK, Okuda K, eds Hepatitis B and C. Carrier to Cancer. India: Elsevier Sciences 268-271.

3. Pyrsopoulus N, Reddy RK (2002) Hepatocellular Carcinoma in Asia. In Sarin SK, Okuda K, eds. Hepatitis B and C. Carrier to Cancer. India: Elsevier Sciences 363-364.

4. Kumar M, Panda D (2014) Role of supportive care for terminal stage hepatocellular carcinoma. J Clin Exp Hepatol 4: S130-139.

5. Mostafa A, Shimakawa Y, Medhat A, Mikhail NN, Chesnais CB, et al. (2016 Excess mortality rate associated with hepatitis $C$ virus infection: A communitybased cohort study in rural Egypt. J Hepatol 64: 1240-1246.

6. Paul SB, Chalamalasetty SB, Vishnubhalta S (2009) Clinical profile, etiology and therapeutic outcome in 324 hepatocellular carcinoma patients at a tertiary care center in India. Oncology 77: 162-171.

7. Kumar R, Saraswat MK, Sharma BC, Sakhuja P, Sarin SK (2008) Characteristics of hepatocellular carcinoma in India: a retrospective analysis of 191 cases. QJM 101: 479-485.

8. Zhu AX (2003) Hepatocellular carcinoma: Are we making progress? Cance Invest 21: 418-428.

9. Cabibbo G, Maida M, Genco C, Parisi P, Peralta M, et al. (2012) Natural history of untreatable hepatocellular carcinoma: A retrospective cohort study. World $\mathrm{J}$ Hepatol 4: 256-261. 
Citation: Ayman A, Azza AH, Yasser K, Kakil R, Jonas F, et al. (2016) The Role of Palliative Care in the Management of Patients with Advanced Hepatocellular Carcinoma: A Single Institution Experience. J Pat Care 2: 112. doi: 10.4172/2573-4598.1000112

Page 5 of 5

10. Cabibbo G, Enea M, Attanasio M (2010) A meta-analysis of survival rates of untreated patients in randomized clinical trials to hepatocellular carcinoma. Hepatology 51: 1274-1283.

11. Al-Kindi SG, Zeinah GF, Hassan AA (2013) Pattern of hospitalization of patients with cancer in an acute palliative care setting: Qatar's experience. Am J Hosp Palliat Care 30: 25-28.

12. Josep M (2012) European Association for the study of the liver, European Organization for Research and Treatment of Cancer: EASL-EORTC Clinical practice guidelines: Management of Hepatocellullar Carcinoma. J Hepatol 56: 908-943

13. Llovet JM, Brú C, Bruix J (1999) Prognosis of hepatocellular carcinoma: The BCLC staging classification. Semin Liver Dis 19: 329-338.

14. Pinato DJ, North BV, Sharma R (2012) A novel externally validated inflammation-based prognostic algorithm in Hepatocellular carcinoma: The prognostic nutritional ((PNI). Br J Cancer 106: 1439-1445.

15. Kim HJ, McGuire DB, Tulman L, Barsevick AM (2005) Symptom clusters: Concept analysis and clinical implications for cancer nursing. Cancer Nurs 28 : 270-282.

16. Ryu E, Kim K, Cho MS, Kwon IG, Kim HS, et al. (2010) Symptom clusters and quality of life in Korean patients with hepatocellular carcinoma. Cancer Nurs 33: $3-10$

17. Miaskowski C, Dodd M, Lee K (2004) Symptom clusters: the new frontier in symptom management research. J Natl Cancer Inst Monogr : 17-21.

18. Lillyman S, Bruce M (2016) Palliative care for people with dementia: A literature review. Int J Palliat Nurs 22: 76-81.

19. Gwilliam B, Keeley V Todd C (2015) Development of prognosis in palliative care study (PiPS) Predictor models to improve prognostication in advanced cancer: prospective cohort study. BMJ Support Palliat Care 4: 390-398.

20. Hui $D$ (2015) Unexpected death in palliative care: What to expect when you are not expecting. Curr Opin Support Palliat Care 9: 369-374
21. Bosch FX, Ribes J, Borràs J (1999) Epidemiology of primary liver cancer Semin Liver Dis 19: 271-285.

22. Fallahian F, Najafi A (2011) Epidemiology of hepatitis $C$ in the Middle East Saudi J Kidney Dis Transpl 22: 1-9.

23. Rasul Kl, Al-Azawi SH, Chandra P (2013) Hepatocellular carcinoma in qatar Gulf J Oncolog 1: 70-75

24. Bruix J, Barrera JM, Calvet X, Ercilla G, Costa J, et al. (1989) Prevalence of antibodies to hepatitis $C$ virus in Spanish patients with hepatocellular carcinoma and hepatic cirrhosis. Lancet 2: 1004-1006.

25. Rodrigo MA, Camila SF, Paulo DN (2013) Hepatocellular carcinoma: The final moments of life. Journal of Cancer Therapy 4: 377-383.

26. Prasad S, Dhiman RK, Duseia A (2007) Lactulose improves cognitive functions and health-related quality of life in patients with cirrhosis who have minimal hepatic encelopathy. Hepatology 45: 549-559.

27. Sharma BC, Sharma P, Agrawal A (2009) Secondary prophylaxis of hepatic encelopathy: An open-label randomized controlled trial of lactulose versus placebo. Gastroenterology 137: 885-891.

28. Lin MH, Wu PY, Tsai ST, Lin CL, Chen TW, et al. (2004) Hospice palliative care for patients with hepatocellular carcinoma in Taiwan. Palliat Med 18: 93-99.

29. Macleod AD (2006) The management of Terminal delirium. Indian J Palliat Care 12: $22-28$

30. Akechi T, Uchitomi Y, Okamura H, Fukue M, Kagaya A, et al. (1996) Usage of haloperidol for delirium in cancer patients. Support Care Cancer 4: 390-392.

31. Sun VC, Sarna L (2008) Symptom management in hepatocellular carcinoma Clin J Oncol Nurs 12: 759-766.

32. Zabora J, BrintzenhofeSzoc K, Curbow B, Hooker C, Piantadosi S (2001) The prevalence of psychological distress by cancer site. Psychooncology 10: 19-28.

33. Mohsen $\mathrm{H}$, Haddad P, Allam A, Hassan A (2014) Patterns in place of cance death in the State of Qatar: A population-based study. PLoS One 9: e109615. 